\title{
Rapid COVID-19 vaccine development
}

\author{
By Barney S. Graham
}

Vaccine Research Center, National Institute of Allergy and Infectious Diseases, National Institutes of Health, Bethesda, MD, USA. Email: bgraham@mail.nih.gov

Finding the fastest pathway to vaccine availability includes the avoidance of safety pitfalls

Rapid development of a vaccine to prevent coronavirus disease 19 (COVID-19) is a global imperative, and defining the stakes and potential hurdles is critical because regulatory and medical decisions are based on benefit:risk calculations. The ability of viruses to achieve pandemic spread is diminished by establishing higher levels of community (herd) immunity, and a key question is whether protection against severe acute respiratory syndrome-coronavirus 2 (SARS-CoV-2) will happen by widespread deployment of an effective vaccine or by repeated waves of infection over the next few years until $\sim 60$ to $70 \%$ of people develop immunity. Because the human population is naïve to SARS-CoV-2, the consequences of repeated epidemics will be unacceptably high mortality, severe economic disruption, and major adjustments to our way of life. Therefore, the benefit of developing an effective vaccine is very high, and even greater if it can be deployed in time to prevent repeated or continuous epidemics.

Vaccine development is usually measured in decades, so having access to approved vaccines available for large-scale distribution before the end of 2020 or even 2021 would be unprecedented. However, new manufacturing platforms, structure-based antigen design, computational biology, protein engineering, and gene synthesis have provided the tools to now make vaccines with speed and precision. Antiviral vaccines can be classified into two broad categories. Genebased vaccines deliver gene sequences that encode protein antigens that are produced by host cells. These include livevirus vaccines, recombinant vaccine vectors, or nucleic acid vaccines. Protein-based vaccines include whole-inactivated virus, individual viral proteins or subdomains, or viral proteins assembled as particles, all of which are manufactured in vitro. Recombinant vaccine vectors and nucleic acid vaccines are best suited for speed because they can be more easily adapted to platform manufacturing technologies in which upstream supply chains and downstream processes are the same for each product. Precision is achieved by knowing the atomic structure of the vaccine antigen and that the targeted epitopes are preserved in the vaccine.

For any vaccine that intends to generate antibody-mediated immunity, delivering a conformationally correct protein is critical. The CoV spike protein is displayed on the virus surface and carries out viral entry. It accomplishes this by undergoing a massive rearrangement that pulls the virus and cell membranes together and fuses them. Therefore, spike is a dynamic and metastable protein that has two major conformational states, prefusion and postfusion. Displaying this antigen so that it maintains the surface contours and chemistry of the original native prefusion spike protein will preserve the epitopes required for eliciting high-quality neutralizing antibody responses. The vaccine formulation and delivery can also be crafted to influence $\mathrm{T}$ cell functions and response patterns. Gene-based delivery can induce $\mathrm{CD} 8^{+} \mathrm{T}$ cells and generally drive a $\mathrm{CD}^{+} \mathrm{T}$ helper 1 cell-type immune response, which has favorable antiviral properties. Adjuvants not only can be used to improve the magnitude and durability of antibody responses induced by protein-based vaccines but can also influence T cell-derived cytokine patterns and thus modulate immune responses.

Safety is a primary goal for vaccines that are given to otherwise healthy people, and there is a risk that vaccination could make subsequent SARS-CoV-2 infection more severe. This has happened before with vaccines based on whole-inactivated virus formulated in alum for a coronavirus of cats and for another unrelated respiratory virus in children. There are two different syndromes previously associated with vaccine-enhanced disease (see the table). One is antibody-dependent enhancement (ADE) (1) and the other is vaccineassociated enhanced respiratory disease (VAERD) (2). ADE is an Fc (the tail end of an antibody)-mediated enhancement of infection typically associated with flaviviruses, such as dengue virus (3). $\mathrm{ADE}$ is measured in vitro on cells that express Fc receptors (FcRs) either naturally or by transfection. The ADE mechanism involves increased binding efficiency of virus-antibody complexes to FcR-bearing cells, which triggers viral entry. This is more likely to occur when vaccine-induced antibody fails to effectively neutralize the virus because of insufficient concentration or affinity or the wrong specificity.

$\mathrm{ADE}$ has been described for feline infectious peritonitis virus (FIPV), a coronavirus that targets macrophages for infection (tropism) and causes a systemic vasculitis-like disease (4). Antibody-mediated disease enhancement was demonstrated after infection in cats that were previously vaccinated with alum-adjuvanted inactivated virus. Although SARS-CoV2 cellular tropism has not been completely defined, it is a respiratory virus, and consistent with coronaviruses that cause Middle East respiratory syndrome (MERS-CoV) and SARS 
(SARS-CoV-1), infection of respiratory epithelium results in a very different pathogenesis than the macrophage-tropic FIPV. ADE was shown to occur for SARS-CoV-1 in vitro in an FcR-bearing human B cell lymphoma cell line and based on detection of viral gene fragments by polymerase chain reaction (PCR). However, there is no experimental data in vivo showing that this type of antibody-mediated entry is relevant to the pathophysiology of respiratory coronaviruses like SARS-CoV-1, and even in vitro, no infectious virus was produced, suggesting an abortive replication cycle (5).

VAERD is a distinct clinical syndrome that occurred in young children in the 1960s when whole-inactivated virus vaccines for measles and respiratory syncytial virus (RSV) were tested $(6,7)$. Immunizing with limiting doses of RSV antigen, especially with conformationally incorrect antigens, can result in two major types of immunological phenomenon that correlate with enhanced respiratory disease (ERD). One is a relatively high ratio of binding antibody to neutralizing antibody. Having a large amount of antibody that binds, but does not neutralize, virus in the presence of a high viral load could potentially result in immune complex deposition and complement activation. This was demonstrated in the small airways of infants during the formalin-inactivated (FI) RSV vaccine trial in 1966 and contributed to inflammation and airway obstruction (8). A similar phenomenon occurred after measles infection of Rhesus macaques that were immunized with whole-inactivated measles virus vaccine (9).

The other observation is that immunization with wholeinactivated virus vaccines followed by RSV infection can result in allergic inflammation (10). Responses that accentuate production of the cytokines interleukin-4 (IL-4), IL-5, and IL13 result in increased mucus production, eosinophil recruitment, airway hyperresponsiveness, and attenuated cytolytic $\mathrm{T}$ cell activity, collectively known as T helper 2 cell $\left(\mathrm{T}_{\mathrm{H}} 2\right) \mathrm{im}$ mune responses. These events potentiate airway dysfunction and delay viral clearance. Therefore, avoiding $\mathrm{T}_{\mathrm{H}} 2$-biased immune responses may be important, especially in young infants with small airways that can be easily obstructed. In the youngest cohort of children who received the FI-RSV vaccine, $80 \%$ of those infected required hospitalization compared with $5 \%$ of placebo recipients (7). In the lung histopathology of the two children who died, there was an abundant polymorphonuclear leukocyte response in lungs that included eosinophils. This is consistent with findings in animal models of $\mathrm{T}_{\mathrm{H}}$ 2-biased $\mathrm{CD}^{+} \mathrm{T}$ cell responses associated with FI-RSV and VAERD (11).

Similar T cell and cytokine response patterns have been shown in mice, cotton rats, cattle, and nonhuman primate models of RSV immunized with whole-inactivated virus formulated with alum (12). In almost all the examples of VAERD demonstrated in humans for RSV and measles, and in animals for SARS (13), the vaccine antigen was whole- inactivated virus. There are caveats to the animal experiments because the allergic inflammation phenomenon can also be elicited by using the same cell line and media to grow the vaccine virus and challenge virus. Cellular components and media additives can cause sensitization to those proteins even without viral antigens present (14).

There are ways to mitigate the risks of vaccine-enhanced disease syndromes informed by prior work on RSV vaccines that should be considered for COVID-19 vaccine development (15). It will be important to demonstrate the potential for vaccine efficacy in early-phase clinical studies by measuring the induction of neutralizing antibodies and in animal models by demonstrating protection against virus replication and disease. Equally important will be using conformationally correct antigens to elicit high-quality, functionally relevant antibody and to avoid induction of non-neutralizing antibody and $\mathrm{T}_{\mathrm{H}} 2$-biased immune responses. Using limiting dilutions of vaccines and examining lung pathology in animals with breakthrough infection after challenge should also help gauge the likelihood of aberrant pathology in vaccinated humans.

Defining the immunological parameters of VAERD in animal models of RSV delayed extensive industry involvement in vaccine development by $\sim 30$ years. Although the potential risk of vaccine-induced antibody or T cell responses leading to adverse responses to natural SARS-CoV-2 infection should be carefully evaluated, there is also a risk of delaying clinical trials in favor of prolonged evaluation of vaccines in animal models that do not fully recapitulate the pathogenesis of disease in humans. In the midst of a pandemic, it is reasonable to require certain qualities in candidate vaccines as described above and to start phase 1 clinical trials based on preliminary immunogenicity in animals and expanded trials based on human immunogenicity and evidence of protection in animal models. Justifying expansion to thousands of subjects in efficacy trials (that is, phase 2 and 3 trials) could include additional evidence of vaccine safety in animals immunized with limiting doses of vaccine and breakthrough infections after SARS-CoV-2 challenge. Judicious evaluation of candidate vaccines in healthy adults in parallel with vaccine studies in animal models and coincident process development to scale-up production capacity provides a path forward with minimal risk to human subjects and the potential for enormous benefit through accelerated COVID-19 vaccine availability.

\section{REFERENCES AND NOTES}

1. J. R. Mascola et al., AIDS Res. Hum. Retroviruses 9, 1175 (1993). doi:10.1089/aid.1993.9.1175 Medline

2. F. P. Polack, Pediatr. Res. 62, 111 (2007). doi:10.1203/PDR.0b013e3180686ce0 Medline

3. K. A. Dowd, T. C. Pierson, Virology 411, 306 (2011). doi:10.1016/j,virol.2010.12.020 Medline

4. C. W. Olsen, W. V. Corapi, C. K. Ngichabe, J. D. Baines, F. W. Scott, J. Virol. 66, 956 (1992). doi:10.1128/JVl.66.2.956-965.1992 Medline

5. M. Jaume et al., J. Virol. 85, 10582 (2011). doi:10.1128/JVI.00671-11 Medline 
6. V. A. Fulginiti, J. J. Eller, A. W. Downie, C. H. Kempe, JAMA 202, 1075 (1967). doi:10.1001/jama.1967.03130250057008 Medline

7. H. W. Kim et al., Am. J. Epidemiol. 89, 422 (1969). doi:10.1093/oxfordjournals.aje.a120955 Medline

8. F. P. Polack et al., J. Exp. Med. 196, 859 (2002). doi:10.1084/jem.20020781 Medline

9. F. P. Polack, S. J. Hoffman, G. Crujeiras, D. E. Griffin, Nat. Med. 9, 1209 (2003). doi:10.1038/nm918 Medline

10. B. S. Graham et al., J. Immunol. 151, 2032 (1993). Medline

11. T. J. Ruckwardt, K. M. Morabito, B. S. Graham, Immunity 51, 429 (2019). doi:10.1016/i.immuni.2019.08.007 Medline

12. A. Muralidharan, C. Li, L. Wang, X. Li, Expert Rev. Vaccines 16, 351 (2017). doi:10.1080/14760584.2017.1260452 Medline

13. M. Bolles et al., J. Virol. 85, 12201 (2011). doi:10.1128/JVI.06048-11 Medline

14. C. A. Shaw et al., Vaccine 31, 306 (2013). doi:10.1016/j.vaccine.2012.11.006 Medline

15. S. K. Browne, J. A. Beeler, J. N. Roberts, Vaccine 38, 101 (2020). doi:10.1016/i.vaccine.2019.10.048 Medline

\section{ACKNOWLEDGMENTS}

Thanks to J. Mascola, K. Morabito, K. Corbett, K. Bok, R. Seder, and M. Kanekiyo for helpful comments and discussions. This work was supported by intramural funding from the National Institute of Allergy and Infectious Diseases, NIH. The author is an inventor on patent applications that involve coronavirus vaccines and monoclonal antibodies.

Published online 8 May 2020

10.1126/science.abb8923 
Potential risks associated with vaccine development for COVID-19

Antibodies that bind virus without neutralizing infectivity can cause disease through increased viral replication or formation of immune complexes that deposit in tissue and activate complement pathways associated with inflammation. Thelper 2 cell $\left(T_{H} 2\right)$-biased responses have also been associated with ineffective vaccines that lead to enhanced disease after subsequent infection. Antibody-dependent enhancement (ADE) of viral replication has occurred in viruses with innate macrophage tropism. Virus-antibody immune complexes and $\mathrm{T}_{\mathrm{H}} 2$-biased responses can both occur in vaccine-associated enhanced respiratory disease (VAERD).

\begin{tabular}{lll} 
& \multicolumn{2}{c}{ Antibody-mediated } \\
\cline { 2 - 3 } & ADE & VAERD \\
\hline Mechanism & $\begin{array}{l}\text { Fc-mediated increase } \\
\text { in viral entry }\end{array}$ & $\begin{array}{l}\text { Immune complex formation and } \\
\text { complement deposition }\end{array}$ \\
\hline Effectors & $\begin{array}{l}\text { Macrophage activation and } \\
\text { inflammatory cytokines }\end{array}$ & $\begin{array}{l}\text { Complement activation and } \\
\text { inflammatory cytokines }\end{array}$ \\
\hline Mitigation & $\begin{array}{l}\text { Conformationally correct antigens and high-quality } \\
\text { neutralizing antibody }\end{array}$
\end{tabular}

T cell-mediated

VAERD

$\mathrm{T}_{\mathrm{H}}$--biased immune

response

Allergic inflammation and $\mathrm{T}_{\mathrm{H}} 2$ cytokines

$T_{H} 1$-biasing immunization and $\mathrm{CD} 8^{+} \mathrm{T}$ cells 